\title{
THE
}

\section{Speech, language, and feeding phenotypes of SATB2-associated syndrome}

\author{
Ashlen Thomason \\ Pankey \\ Beth Nutt \\ Aisling R. Caffrey \\ University of Rhode Island, aisling_caffrey@uri.edu
}

Yuri A. Zarate

Follow this and additional works at: https://digitalcommons.uri.edu/php_facpubs

The University of Rhode Island Faculty have made this article openly available.

Please let us know how Open Access to this research benefits you.

This is a pre-publication author manuscript of the final, published article.

Terms of Use

This article is made available under the terms and conditions applicable towards Open Access

Policy Articles, as set forth in our Terms of Use.

\section{Citation/Publisher Attribution}

Thomason, A., Pankey, E., Nutt, B., Caffrey, A. R., \& Zarate, Y. A. Speech, language, and feeding phenotypes of SATB2-associated syndrome. Clin Genet., 96(6), 1- 8. doi: 10.1111/cge.13619

Available at: https://doi.org/10.1111/cge.13619 


\section{Speech, Language, and Feeding Phenotypes of SATB2-associated syndrome}

Running title: Language and feeding in SATB2-associated syndrome

Ashlen Thomason ${ }^{1}$, Emily Pankey ${ }^{1}$, Beth Nutt ${ }^{1}$, Aisling R. Caffrey ${ }^{2}$, Yuri A. Zarate ${ }^{3}$

${ }^{1}$ Audiology/Speech Pathology Department, Arkansas Children's Hospital, Little Rock, AR, USA

${ }^{2}$ Health Outcomes, College of Pharmacy, University of Rhode Island, Kingston, RI, USA

${ }^{3}$ Section of Genetics and Metabolism, University of Arkansas for Medical Sciences, Little Rock, AR, USA

\section{Acknowledgements}

The authors would like to sincerely thank the families for their participation and continued support. The authors would also like to acknowledge all of the departments and volunteers at Arkansas Children's Hospital for their help with the International SATB2-associated syndrome clinics.

\section{Conflicts of interest}

The authors have no conflicts of interest to disclose

\section{Data availability statement}

The data that support the findings of this study are available from the corresponding author upon reasonable request. 


\begin{abstract}
SATB2-associated syndrome (SAS) is a recently-identified disorder characterized by neurodevelopmental deficits and craniofacial anomalies. Assessments of speech, language, and feeding-related issues were conducted among 61 individuals with SAS (median age=86 months, range $=26$ months to 29 years of age). Individuals with SAS were mostly non-verbal communicators $(72.1 \%)$ with severe deficits in both language comprehension and expression. The majority of individuals had receptive vocabulary skills of a child younger than 3-years of age. Based on parent report the average spoken lexicon was $28.6(\mathrm{sd}=84.6)(\mathrm{n}=55)$ with a range of 0 to 500 (median=5 words). All of the individuals with SAS with enough verbal ability either showed signs of childhood apraxia of speech or already had a diagnosis $(n=40)$ and $73.3 \%$ exhibited problems with reliable communication with unfamiliar partners. Hypernasal resonance (17.8\%) due to velopharyngeal insufficiency secondary to a history of cleft palate and/or apraxic palatal movement (60.0\% of hypernasal patients with no history of cleft palate), problems with chewing (68.2\%), overstuffing the mouth with solids (64.9\%), pharyngeal phase dysphagia $(60.8 \%)$, and sialorrhea $(63.3 \%)$ were common in this population. Mutation type was not predictive of receptive or expressive language abilities. We developed language and communication treatment recommendations based on these findings.
\end{abstract}

Keywords: SATB2, Speech, Language, Feeding 


\section{Introduction}

SATB2- associated syndrome (SAS) is a multisystem disorder caused by alterations of the SATB2 gene manifesting as craniofacial anomalies and neurodevelopmental problems warranting multidisciplinary assessment and management. ${ }^{1-3}$ The reported craniofacial phenotype in SAS includes facial dysmorphic features, palatal abnormalities (cleft palate, high-arched palate, submucous cleft palate, bifid uvula), micrognathia, and several dental anomalies. ${ }^{1,2,4}$ Neurodevelopmental problems among individuals with SAS are characterized by intellectual disability, global developmental delay, limited or absent verbal speech, behavioral issues (autistic-like features, jovial/overly-social personality), epilepsy, and feeding difficulties. ${ }^{4}$

Verbal communication is the area of development most severely impacted in SAS ${ }^{3,4}$. Previous reports indicate that affected individuals often demonstrate significant impairment of verbal communication abilities, receptive and language deficits, reliance on augmentative and alternative communication (AAC) modalities, features potentially consistent with apraxia of speech, and hallmarks of an autism spectrum disorder. ${ }^{1,2,4-13}$

While the development of receptive language abilities beyond those of expressive language have been reported in several individuals, the degree of verbal impairment is reported to be variable, ranging from absent or near absent to more developed, yet still substantially delayed. ${ }^{3,4}$ Furthermore, other comorbid palatal anomalies and feeding difficulties that can have an impact of speech production, have been reported to commonly occur in SAS. ${ }^{1,2,4-15}$

We here present the first report of speech, language, and feeding difficulties conducted using direct assessments and screening procedures in a large cohort of individuals with SAS from different demographic and genetic backgrounds. Data for progression of skills is presented 
and genotype-phenotype correlations on speech outcomes explored. Based on our results, we provide additional language and feeding recommendations for care of individuals with SAS. This information can be used to inform treating clinicians and families of individuals with SAS about expectations and best practices.

\section{Subjects and Methods}

\section{Subjects}

As part of two separate multidisciplinary international clinics which took place in 2017 and 2018, individuals with SAS were evaluated by the following specialties: genetics, speech, audiology, otolaryngology, dentistry, orthodontics, maxillofacial surgery, and psychiatry. All individuals were confirmed to have the diagnosis of SAS and their medical records were reviewed, including prior cognitive evaluations, when available. Based on a literature review of the known speech-language and feeding characteristics of individuals with SAS, speech-language pathologists (SLPs) evaluated the domains of receptive language, expressive language, speech production, resonance, and feeding using subtests from standardized speech tests, criterionreferenced speech screening tools, rubric-based subjective scales, clinician observations, and parent interview. Evaluations were conducted in English. As subtests and criterion-referenced items were standardized on English-speakers, data from individuals whose primary language was not English could not be used for those assessments.

\section{Molecular studies}

Except for a single individual (SATB2-85), remaining participants were included in previous publications detailing their clinical and genetic investigations. ${ }^{3,4,8,9}$ Individual SATB285 underwent molecular cytogenetic studies with an array CGH+SNP (4x180, Agilent 
Technologies, Santa Clara, CA). Underlying molecular alterations were grouped for analysis as follows: large chromosomal deletions or duplications (2q33.1 deletions and duplications encompassing SATB2 and other contiguous genes), truncating pathogenic variants (predicted nonsense and frameshift), intragenic deletions, canonical splice site, and missense variants. All families reported herein agreed to share clinical information and were enrolled under a research clinical registry protocol approved by the Institutional Review Board of the University of Arkansas for Medical Sciences.

\section{Receptive language and communication skills}

Each individual's receptive language abilities were assessed using the Vocabulary subtest of the Test for Auditory Comprehension of Language-Fourth Edition (TACL-4) 16 (Supplementary materials and methods). The TACL-4 scaled subtest was interpreted as average (8-13), low average (7), mild deficit (6), moderate deficit (5), or severe deficit (1-4). Overall communication abilities were rated using the Communication Function Classification System (CFCS). ${ }^{17}$ The $C F C S$ provides 5 levels (CFCS I, II, III, IV, V) to describe everyday communication performance with sending and receiving messages via any modality (e.g. spoken language, sign language, speech-generating electronic devices, etc.) with familiar and unfamiliar communication partners. Individuals with SAS were rated on the CFCS scale based on clinical judgment of overall language abilities using any communication modality.

Speech Intelligibility Individuals who had the verbal ability to imitate words were administered the LinguiSystems Articulation Test (LAT) - Apraxia Screening Summary. ${ }^{18}$ The LAT Apraxia Screening Summary is based on the child's sequential productions of twelve multisyllabic words across three trials. Inconsistent repetition of a multisyllabic word, a hallmark sign of Childhood Apraxia of Speech (CAS), has been previously assessed as a screening tool in 
other genetic conditions. ${ }^{19-21}$ Evaluating clinicians phonetically transcribed utterances clinically judged to be in known context (e.g. a child pointed to his sister named Jenna and produced an utterance with similar phonetic similarity such as "Zin-uh") and compared those phonetically transcribed words to the target word. Clinicians analyzed the error patterns in the transcribed utterances. The analysis of these error patterns included consideration for characteristics of a phonological delay, an articulation deficit, and CAS. Signs of CAS, including vowel errors, idiosyncratic errors, and prosody differences were a basis to form a clinical impression of CAS characteristics.

\section{Resonance}

Among individuals determined to be verbal communicators, with the verbal speaking abilities to imitate words, clinicians evaluated each patient's resonance. The following perceptual 11 point scale was used by the evaluating SLPs to rate resonance: -5 , severely hyponasal; -4 , moderately-severely hyponasal; -3 , moderately hyponasal; -2 , mildly-moderately hyponasal; -1 , mildly hyponasal; 0 , within normal limits; 1 , mildly hypernasal; 2 , mildly-moderately hypernasal; 3, moderately hypernasal; 4, moderately to severely- hyponasal; 5 , severely hyponasal. Individuals with components of both hypernasality and hyponasality were listed as having mixed resonance with two separate numeric ratings for both hypernasality and hyponasality. SLPs noted the presence or absence of nasal air emission (NAE): visible NAE assessed through use of detail reflector mirror, audible NAE assessed perceptually by clinical assessment of speech productions, or both. Clinicians also noted clinical signs of velopharyngeal insufficiency. Medical records were reviewed to identify a history of cleft palate or submucous cleft palate.

\section{Feeding, language acquisition, expressive vocabulary, modes of communication}


Evaluating SLPs developed a SAS-specific parent interview questionnaire to assess language acquisition, expressive vocabulary, and modes of communication (Supplementary materials and methods). When parents provided an estimated range, rather than a whole-number estimation, the median value was used for data analysis (e.g. parent estimation of " 10 to 20 words" was given a value of 15 words). Additionally, evaluating SLPs noted clinical impressions regarding the mean length of utterance used during the session. SLPs categorized individuals as either primarily verbal or non-verbal under the following criteria: 1) primarily non-verbal, reportedly uses no more than 10 words in a day and uses non-verbal communication more often than verbal, or 2) primarily verbal, reportedly uses more than 10 words in a day and uses speaking as primary mode of communication. External medical records were also reviewed to supplement information obtained from the clinic visit and parental report in all cases.

\section{Statistics}

Demographics and clinical characteristics of speech, language, and feeding were assessed as categorical (number, percent) or continuous measures (mean, standard deviation [sd]; median, interquartile range [IQR], range). Chi-square, Fisher's exact, or t-tests were used, as appropriate, to quantify the impact of molecular alterations on speech outcomes (e.g. genotype-phenotype associations).

\section{Results}

Baseline demographic and clinic characteristic are summarized in table 1 while results of screening procedures are described in table 2. Sixty-one individuals (31 males, 50.8\%) of different ages (median=86 months, range $=26$ months to 29 years of age), with a variety of molecular alterations (42.6\% with truncating variants), from seven different countries $(88.5 \%$ from the United States) were evaluated during two separate clinics ( $\mathrm{n}=9,14.8 \%$ were evaluated 
twice). The most common primary mode of communication was gestures (59.0\%), either alone or in combination with signs and/or AAC while verbal communication was primarily used by 14 (22.9\%) individuals. Cognitive evaluations had been completed for 11 individuals (range: 3478). Supplementary Table 1 features speech production, language, cognitive, and feeding data gathered for each individual in this cohort. Supplementary tables 2 and 3 detailed molecular results for each individual.

\section{Receptive and expressive language skills are significantly affected in individuals with SAS}

The average raw score for the TACL-4 Vocabulary subtest was $10.1(\mathrm{n}=60, \mathrm{sd}=10.7)$; a raw score below 15 has an age equivalent of less than 3-years 0-months for this subtest (Figure 1). Scores ranged from 0 to 38 (the highest scoring individual being a 13-year old having an age equivalent of 5-years 9 -months for this subtest), with 17 individuals (29\%) scoring 0 by not correctly identifying any receptive vocabulary words (e.g. home, box, girl, drink). Scaled TACL4 Vocabulary subtest scores were also reflective of significant impairment $($ mean=2.0,sd=1.6) with $76.8 \%(43 / 56)$ below $<1^{\text {st }}$ centile rank (Figure 1).

Of the 61 individuals with SAS assessed, $72.1 \%(n=44)$ were primarily nonverbal. The average age of reported first words was 27.6 months $(\mathrm{sd}=14.4)$ with a range from 8-months to 66-months ( $n=44$, only includes individuals who have spoken first words). Based on parent report the average spoken lexicon of individuals with SAS ( $\mathrm{n}=55)$ was $28.6(\mathrm{sd}=84.6)$ words, with a range of 0 to 500 (median=5 words) (Figure 1). However, this average includes three individuals with estimated spoken vocabularies of 200, 350, and 500 words. Excluding those three individuals with markedly higher expressive language abilities than other individuals with SAS, the average fell to 10 words ( $\mathrm{sd}=16.2$; median=4.3). The remaining 52 individuals had an average expressive lexicon of 2.5 words for children under 3 -years of age, 6.2 words for children 
3-years 1-month through 6-years 0-months, 13.9 words for children 6-years 1-month through 12years 0-months, and 10.2 words for those 12-years 1-month through 29-years of age. For reference, 20 words in a child's spoken vocabulary is typically achieved by 18 -months of age and 200 words by 24 -months of age ${ }^{20}$. By caregiver report, $16 \%$ of individuals tested had no spoken vocabulary words. Individuals with large deletions, truncating mutations, missense mutations, and intragenic deletions were all represented among individuals with no spoken vocabulary words (Table 2). The "highest functioning" children evaluated in this cohort were an 8-year 0-month old boy (SATB2\#53) with an estimated spoken vocabulary of 200 words who

receives >120 minutes of speech therapy per week, a 5-year 6-month-old boy (SATB2\#004) with an estimated spoken vocabulary of 350 words who receives 120 minutes of speech therapy per week, and a 13-year 4-month old girl (SATB2\#51) with an estimated spoken vocabulary of 500 words who receives 60-90 minutes of speech therapy per week.

Mean length of utterance (MLU) among 7 individuals who were classified as primarily verbal communicators was 2.39 words, ranging from 1.00 to 6.75 . However, that utterance length drops to 1.67 words per utterance when omitting a high-functioning individual with an outlier MLU of 6.75. For reference, a MLU of 1.75 is typically reached by 26-months of age ${ }^{22}$.

\section{Most individuals with SAS have difficulties with everyday communication}

On the CFCS, $73.3 \%(n=44)$ individuals exhibited problems with reliable communication with unfamiliar partners (CFCS III, IV, or V). Of the 60 individuals rated, 10 individuals (16.6\%) were rated Level V (Seldom Effective Sender and Receiver even with familiar partners). Apraxia of speech is common in SAS

Forty individuals with SAS had enough verbal ability for noting clinical characteristics of apraxia. Of these, 31 (77.5\%) had received a prior diagnosis of CAS (22 also judged to have 
characteristics of CAS during the clinic visit, 8 did not produce enough verbal utterances in known context for an adequate analysis, and 1 not screened). Nine additional individuals (22.5\%) were judged to exhibit CAS based on hallmark characteristics noted by the SLP. The LATApraxia Screening Summary ${ }^{22}$ results revealed very low ability for consistently repeating multisyllabic words among 8 participants who were primarily verbal. The average number of words repeated consistently (not necessarily correctly) was 6.37 (ranging from 4 to 10), which was highly indicative of CAS.

\section{Oral-motor and resonance issues are prevalent in this population}

For individuals with SAS with enough verbal output for making a perceptual resonance judgment $(n=28), 82.1 \%(n=23)$ had normal resonance, $7.1 \%(n=2)$ had perceptually mild hypernasal resonance, and $10.7 \%(n=3)$ had perceptually moderate hypernasal resonance during speech. There was no history of cleft palate in $60.0 \%(n=3)$ of individuals noted to have any hypernasal resonance. A history of cleft palate was noted with $36.1 \%(n=22)$ of individuals with SAS $(n=61)$.

A history of sialorrhea was reported in $63.3 \%$ ( $n=38$ of 60 assessed) of individuals with SAS, with resolution reported by an average of 48 months (range of drooling cessation: 2.5 to 7 -years of age). Among 24 individuals (40.0\%), sialorrhea persisted beyond 48-months of age, though 41.7\% ( $\mathrm{n}=10)$ reported significant improvements over time. Regarding feeding difficulties, $64.9 \%(n=37$ of 57 assessed) of individuals with SAS analyzed overstuffed their mouths during meals. Of the 22 individuals whose caregivers were asked about chewing, $68.2 \%(n=15)$ reported difficulty with chewing. While $60.8 \%$ ( $n=14$ of 23 assessed) of individuals SAS had a reported history of pharyngeal dysphagia, $61.6 \%$ ( $n=37$ of 60 assessed) of caregivers reported no present signs of aspiration. 


\section{Speech progression over time}

Nine individuals were seen in consecutive evaluations a year apart. All but two (77.8\%) individuals made gains in his or her receptive vocabulary raw score with an average net gain of 3.8 words (ranging from five fewer words to a gain of 19 words). Despite gains in raw scores, the net scaled score changed by -0.33 (ranging from a drop in 1 scaled score to no gain); reflecting slower acquisition of vocabulary compared to the rate of same-age peers. Likewise, spoken vocabulary grew in four of the nine $(44.4 \%)$ returning individuals. The average net gain was 29.2 words (sd=82.9) among the 9 individuals. However, the average fell to 1.62 words $(\mathrm{sd}=5.7)$ when assessed without the one individual who gained over 250 words in the year between visits. From year-to-year, among those without gains, two had the same number of words, two more regressed from one spoken word to zero, and the last one regressed from 10 words to 2-3 words. Two of those three (66.7\%) individuals who regressed in spoken vocabulary had autism or were clinically suspected as having an autism spectrum disorder.

\section{Genotype-phenotype correlations}

Overall, mutation type was not predictive of clinical features or language development (Table 2). Proportion of non-verbal individuals, mean $T A C L-4$ raw and scaled scores, number of individuals with $T A C L-4$ receptive age equivalent younger than 3 years of age, mean reported age at first words, mean CFCS level, and frequency of CAS diagnosis did not show statistical differences between molecular groups. Individuals with intragenic deletions did have a lower mean reported number of spoken words $(1.3 \pm 1.9)$ compared with other groups $(\mathrm{p}=0.02)$ (Figure 1).

\section{Discussion}


This study represents the first direct investigation of the impact of SAS on receptive language, expressive language, speech production, resonance, and feeding. Based on our findings and experience with this cohort of individuals, we present a summary of main findings along with recommendations for speech, language, resonance, and feeding below, also summarized in Table 3.

Individuals with SAS largely have significant deficits in receptive vocabulary skills relative to age. The majority of individuals had receptive vocabulary skills of a child younger than 3-years of age ${ }^{16}$. As a cohort, individuals' receptive vocabulary scaled scores were progressively less than those of same-age peers with less accelerated growth over time than neurotypical children. Despite these deficits, as the cohort aged, raw scores for receptive vocabulary skills improved over time for most. For those individuals that were seen on two occasions, we were able to document net improvement in receptive skills. Given the trend of receptive vocabulary growth across age and the improvement displayed by most returning participants after one year, SLPs treating individuals with SAS should continually target receptive vocabulary skills. As $29 \%$ of individuals with SAS exhibited scores of 0 in their $T A C L$ 4 subtest scaled scores, managing SLPs may not be able to measure receptive language growth through pointing of pictures from an array presented. Non-verbal IQ testing should be considered for all individuals with SAS for educational planning to factor out the negative impact of a child's language impairment when assessing their cognitive profile.

Most individuals with SAS will not use spoken language as their primarily means of communication. With an average spoken lexicon of 28.6 words and average MLU for verbal participants of 1.67, individuals with SAS should be considered for AAC to enhance their expressive abilities. Presenting AAC earlier and at home and school, with appropriate training in 
those settings, may improve expressive vocabulary growth. Mutation type was not predictive of speech outcomes. Several individuals with SAS are inconsistent senders and receivers of information with familiar partners and even greater number have problems communicating with unfamiliar partners. ${ }^{17}$ Targeted use of AAC to enable or augment communication should be implemented with a progressively growing circle of communication partners, and training of those communication partners as needed.

Essentially all children with enough verbal ability to screen informally with a criterion reference tool exhibited characteristics of CAS. ${ }^{19,20}$ Managing SLPs should assume that children with SAS will have motor planning deficits associated with apraxia as they develop verbal speech. Clinicians should target speech production with a motor-planning approach, not waiting until the child has enough verbal ability to corroborate an apraxia diagnosis with formal testing. A single randomized control trial of apraxia treatment approaches comparing the Nuffield Dyspraxia Programme-3 and the Rapid Syllable Transitions Treatment determined that when these interventions are delivered in intensive one-hour sessions four days per week across three weeks, patients demonstrate comparable gains in word accuracy one month after treatment with either therapy approach. ${ }^{23}$ For individuals with CAS, higher dose or higher-dose frequency of intervention results in better outcomes than lower dose or dose frequency of those same interventions ${ }^{24}$ Individual therapy is recommended as it yields a higher frequency of responses. ${ }^{24}$ It is of note that therapy intensity is comprised of many complex variables, including dose, dose form, dose frequency, session duration, and total intervention duration; therapy intensity and patient outcomes are not always positively correlated, and treatment intensity can reach a point of diminishing return. ${ }^{25}$ Intellectual disabilities and attention difficulties common in SAS, may 
limit therapy dosage (number of responses per session) and sustained attention for long session durations.

Mild to moderate hypernasality was present in $18 \%$ of individuals and independent of a history of cleft palate. Resonance, particularly for hypernasality, should be monitored. The source of velopharyngeal insufficiency could be related to a history of cleft palate, mistiming of velopharyngeal closure secondary to motor planning deficits, or a co-occurrence of both. A referral to an otolaryngologist for nasendoscopy of assessment of velopharyngeal insufficiency should be made if clinicians perceive hypernasality and/or nasal air emission (not phonemespecific nasal air emission). Difficulty with chewing (68\%) and overstuffing the mouth with solids $(65 \%)$ were common among children with SAS; thus, feeding therapy may be warranted as part of the individual's plan of care. We recommend monitoring for signs and symptoms of aspiration, as there is a high incidence of pharyngeal dysphagia (61\%); a formal swallow study may be warranted to rule out aspiration and to determine if a patient needs compensatory strategies or thickened liquids to protect a child's respiratory health. Lastly, while sialorrhea is common for individuals with SAS, it seems to improve over time.

This study has several limitations and the results should be interpreted with some caution. While the sample size (61 individuals) is the largest cohort objectively analyzed for speech and language deficits, a larger prospective cohort is desirable to validate these results. Although all individuals have the diagnosis of SAS, different molecular alterations that resulted in this syndrome were included and some differences could be the result of other modifier genes and the impact of affected contiguous genes for those with large deletions and duplications was not analyzed. . Likewise, if the language phenotype seen in this population is commensurate with the individual's general intellectual or functioning level could not be evaluated. Detailed cognitive 
evaluations, while often attempted, could not yield a reliable IQ score to be reported in the majority of patients. Individuals from different countries of origin were included and language barriers could have contributed to reduced validity of results given via parent report. Speech and language outcomes could also have been impacted by hearing impairment in this cohort although no history of hearing loss was recorded for any of the individuals evaluated. The presence of articulation deficits and/or phonological delay and dysarthria of the velopharyngeal port could not be ruled out as a contributing or co-occurring factor to explain some of the results reported. Lastly, some of the information obtained for this study was based on parental recollection. To reduce error in the data, medical records were reviewed for all individuals. In summary, individuals with SAS have a plethora of language, speech production, resonance, and feeding deficits that go beyond the primarily reported speech delay. These difficulties can be pervasive and necessitate a comprehensive, ongoing, multidisciplinary approach. The language and communication treatment recommendations were developed based on the results of this study to assist treating clinicians and caregivers of individuals with SAS in developing an effective treatment plan. 


\section{References}

1. Zarate YA, Fish JL. SATB2-associated syndrome: Mechanisms, phenotype, and practical recommendations. Am J Med Genet A. 2017;173:327-337.

2. Zarate YA, Kaylor J, Fish J. SATB2-Associated Syndrome. In: Adam MP, Ardinger HH, Pagon RA, et al., eds. GeneReviews(R). Seattle (WA)1993.

3. Zarate YA, Bosanko KA, Caffrey AR, et al. Mutation update for the SATB2 gene. Hum Mutat. 2019.

4. Zarate YA, Smith-Hicks CL, Greene C, et al. Natural history and genotype-phenotype correlations in 72 individuals with SATB2-associated syndrome. Am J Med Genet A. 2018;176:925-935.

5. Bengani H, Handley M, Alvi M, et al. Clinical and molecular consequences of diseaseassociated de novo mutations in SATB2. Genet Med. 2017;19:900-908.

6. Leoyklang P, Suphapeetiporn K, Siriwan P, et al. Heterozygous nonsense mutation SATB2 associated with cleft palate, osteoporosis, and cognitive defects. Hum Mutat. 2007;28:732-738.

7. Rosenfeld JA, Ballif BC, Lucas A, et al. Small deletions of SATB2 cause some of the clinical features of the 2q33.1 microdeletion syndrome. PLoS One. 2009;4:e6568.

8. Zarate YA, Kalsner L, Basinger A, et al. Genotype and phenotype in 12 additional individuals with SATB2-associated syndrome. Clin Genet. 2017;92:423-429.

9. Zarate YA, Perry H, Ben-Omran T, et al. Further supporting evidence for the SATB2associated syndrome found through whole exome sequencing. Am J Med Genet A. 2015;167A:1026-1032. 
10. Balasubramanian M, Smith K, Basel-Vanagaite L, et al. Case series: 2q33.1 microdeletion syndrome--further delineation of the phenotype. J Med Genet. 2011;48:290-298.

11. Docker D, Schubach M, Menzel M, et al. Further delineation of the SATB2 phenotype. Eur J Hum Genet. 2014;22:1034-1039.

12. Kaiser AS, Maas B, Wolff A, et al. Characterization of the first intragenic SATB2 duplication in a girl with intellectual disability, nearly absent speech and suspected hypodontia. Eur J Hum Genet. 2015;23:704-707.

13. Lee JS, Yoo Y, Lim BC, Kim KJ, Choi M, Chae JH. SATB2-associated syndrome presenting with Rett-like phenotypes. Clin Genet. 2016;89:728-732.

14. FitzPatrick DR, Carr IM, McLaren L, et al. Identification of SATB2 as the cleft palate gene on 2q32-q33. Hum Mol Genet. 2003;12:2491-2501.

15. Van Buggenhout G, Van Ravenswaaij-Arts C, Mc Maas N, et al. The del(2)(q32.2q33) deletion syndrome defined by clinical and molecular characterization of four patients. Eur J Med Genet. 2005;48:276-289.

16. Carrow-Woolfolk E. Test for Auditory Comprehension of Language-Fourth Edition. In: Pro-Ed: Austin, TX; 2014.

17. Hidecker MJ, Paneth N, Rosenbaum PL, et al. Developing and validating the Communication Function Classification System for individuals with cerebral palsy. Dev Med Child Neurol. 2011;53:704-710.

18. Bowers L HR. LinguiSystems Articulation Test (LAT) - Apraxia Screening Summary. In: East Moline, IL: LinguiSystems; 2010. 
19. Teverovsky EG, Bickel JO, Feldman HM. Functional characteristics of children diagnosed with Childhood Apraxia of Speech. Disabil Rehabil. 2009;31:94-102.

20. Nicolosi L HE, Kresheck J. Terminology of communication disorders 4th Edtion ed: Williams \& Wilkins; 2006.

21. Mei C, Fedorenko E, Amor DJ, et al. Deep phenotyping of speech and language skills in individuals with 16p11.2 deletion. Eur J Hum Genet. 2018;26:676-686.

22. Brown R. A first language: The early stages. Harvard University Press; 1973.

23. Morgan AT, Murray E, Liegeois FJ. Interventions for childhood apraxia of speech. Cochrane Database Syst Rev. 2018;5:CD006278.

24. Kaipa R, Peterson AM. A systematic review of treatment intensity in speech disorders. Int J Speech Lang Pathol. 2016;18:507-520.

25. Baker E. Optimal intervention intensity in speech-language pathology: discoveries, challenges, and unchartered territories. Int J Speech Lang Pathol. 2012;14:478-485. 


\section{Figure legends}

Figure 1. A., B. TACL-4 raw and scaled scores by age; C., D. Reported lexicon median with interquartile range by age and molecular alteration. 